\title{
Study of the common genetic background for rheumatoid arthritis and systemic lupus erythematosus
}

\author{
Gisela Orozco, ${ }^{1}$ Steve Eyre, ${ }^{1}$ Anne Hinks, ${ }^{1}$ John Bowes, ${ }^{1}$ Ann W Morgan, ${ }^{2}$ \\ Anthony G Wilson, ${ }^{3}$ Paul Wordsworth, ${ }^{4}$ Sophia Steer, ${ }^{5}$ Lynne Hocking, ${ }^{6}$ UKRAG \\ consortium, Wendy Thomson, ${ }^{1}$ Jane Worthington, ${ }^{1}$ Anne Barton ${ }^{1}$
}

${ }^{1}$ Arthritis Research UK

Epidemiology Unit, Manchester Academic Health Science Centre, The University of Manchester, Manchester, UK ${ }^{2}$ NIHR-Leeds Musculoskeletal Biomedical Research Unit, Leeds Institute of Molecular Medicine, Leeds, UK ${ }^{3}$ School of Medicine and Biomedical Sciences, The University of Sheffield, Sheffield, UK

${ }^{4}$ Botnar Research Centre, University of Oxford Institute of Musculoskeletal Sciences, Oxford, UK

${ }^{5}$ Clinical and Academic Rheumatology, King's College Hospital NHS Foundation Trust, London, UK

${ }^{6}$ Bone Research Group, Department of Medicine and Therapeutics, University of Aberdeen, Aberdeen, UK

\section{Correspondence to}

Gisela Orozco, Arthritis Research UK Epidemiology Unit, Manchester Academic Health Science Centre, The University of Manchester, Stopford Building, Manchester M13 9PT, UK

gisela.orozco@manchester. ac.uk

GO and SE contributed equally to this work.

Accepted 3 October 2010

Published Online First

10 November 2010

\section{ABSTRACT}

Background Evidence is beginning to emerge that there may be susceptibility loci for rheumatoid arthritis (RA) and systemic lupus erythematosus (SLE) that are common to both diseases.

Objective To investigate single nucleotide polymorphisms that have been reported to be associated with SLE in a UK cohort of patients with RA and controls.

Methods 3962 patients with RA and 9275 controls were included in the study. Eleven SNPs mapping to confirmed SLE loci were investigated. These mapped to the TNFSF4, BANK1, TNIP1, PTTG1, UHRF1BP1, ATG5, JAZF1, BLK, KIAA1542, ITGAM and UBE2L3 loci. Genotype frequencies were compared between patients with RA and controls using the trend test.

Results The SNPS mapping to the BLK and UBE2L3 loci showed significant evidence for association with RA. Two other SNPs, mapping to ATG5 and KIAA1542, showed nominal evidence for association with RA ( $p=0.02$ and $p=0.02$, respectively) but these were not significant after applying a Bonferroni correction. Additionally, a significant global enrichment in carriage of SLE alleles in patients with RA compared with controls $\left(p=9.1 \times 10^{-7}\right)$ was found. Meta-analysis of this and previous studies confirmed the association of the $B L K$ and UBE2L3 gene with RA at genome-wide significance levels $\left(p<5 \times 10^{-8}\right)$. Together, the authors estimate that the SLE and RA overlapping loci, excluding HLA-DRB1 alleles, identified so far explain $\sim 5.8 \%$ of the genetic susceptibility to RA as a whole.

Conclusion The findings confirm the association of the $B L K$ and UBE2L3 loci with RA, thus adding to the list of loci showing overlap between RA and SLE.

\section{INTRODUCTION}

Rheumatoid arthritis (RA) and systemic lupus erythematosus (SLE) are both autoimmune rheumatological diseases thought to have a substantial genetic contribution to susceptibility. ${ }^{1}{ }^{2}$ Recent genome-wide association studies (GWAS) in both diseases have disclosed a number of genetic loci that that are commonly associated. Examples include the human leucocyte antigen (HLA) locus, the R620W (rs2476601) polymorphism within the PTPN22 gene and association with the chromosome 6q23/TNFAIP3 locus. ${ }^{3} 4$ The high degree of familial aggregation that has been shown for RA and SLE further supports the presence of common genetic risk factors for both diseases. 56
Recent GWAS in SLE have identified a number of novel associations, some of which have not been previously investigated in RA. ${ }^{4-10}$ We have reported previously on the overlap between type 1 diabetes and RA susceptibility loci and there are now numerous examples of overlap between diverse autoimmune diseases. ${ }^{11-14} \mathrm{We}$, therefore, hypothesised that the underlying autoimmunity of SLE and RA may be underpinned by additional overlap in the genetic susceptibility loci. The aim of this work was to investigate whether single nucleotide polymorphism (SNP) markers that had been reported to be associated with SLE were also associated with RA in a UK population of patients with RA and controls.

\section{METHODS}

\section{Samples}

Three thousand nine hundred and sixty-two patients with RA and 9275 controls were included in the study. The patients with RA were recruited as described previously ${ }^{15}$ and all satisfied the 1987 American College of Rheumatology criteria for RA modified for genetic studies. ${ }^{16} 17$ The clinical characteristics of the cohort have been described previously, but briefly, $71.8 \%$ were female, $72.1 \%$ were positive for rheumatoid factor and $69.7 \%$ positive for anticyclic citrullinated peptide (anti-CCP) antibodies. All samples were collected with ethical committee approval (MREC 99/8/84) and all individuals provided informed consent.

\section{SNP selection and genotyping}

A panel of 15 autosomal SNPs was selected for investigation from a recent large-scale replication study of SLE-associated loci, ${ }^{10}$ and proxy SNPs were included where assays could not be designed for the original SNP tested.

Of the 21 SLE loci identified to date, we have previously reported association with PTPN22, STAT4 and the 6q23/TNFAIP3 locus. ${ }^{15}$ 18-20 The association of HLA-DRB1 alleles with RA has been extensively studied in the past. Different SNPs mapping to the FCGR2A gene (rs12746613; $\mathrm{r}^{2}=0.19$ with the SLE SNP rs1801274) and PRDM1 (rs548234, $\mathrm{r}^{2}=0.07$ with the SLE SNP rs2245214) have been reported to be associated with RA in meta-analysis including the samples tested in the current cohort. ${ }^{21}$ SNPs mapping to the remaining 15 loci were selected for investigation. However, four SNPs mapping to IRF5, IRAK1, PXK and IL10 either failed assay 
Table 1 Association results for SLE risk variants genotyped in UK RA cases and controls

\begin{tabular}{|c|c|c|c|c|c|c|c|c|c|}
\hline Chr & Locus & SNP & $\begin{array}{l}\text { Major allele/ } \\
\text { minor allele }\end{array}$ & MAF cases & MAF controls & HWE controls & $\begin{array}{l}\text { Trend test } \\
\text { p value }\end{array}$ & $\begin{array}{l}\text { Corrected } \\
\text { p value }\end{array}$ & Allelic OR (95\% Cl) \\
\hline 1 & TNFSF4 & rs10489265 & $\mathrm{T} / \mathrm{G}$ & 0.25 & 0.24 & 0.56 & 0.11 & & $1.05(0.99$ to 1.12$)$ \\
\hline 4 & BANK1 & rs10516487 & $\mathrm{C} / \mathrm{T}$ & 0.31 & 0.31 & 0.66 & 0.52 & & $0.98(0.93$ to 1.04$)$ \\
\hline 5 & TNIP1 & rs10036748 & $\mathrm{C} / \mathrm{T}$ & 0.24 & 0.24 & 0.21 & 0.46 & & $1.02(0.96$ to 1.09$)$ \\
\hline 5 & PTTG1 & rs2431697 & $\mathrm{T} / \mathrm{C}$ & 0.42 & 0.43 & 0.62 & 0.06 & & $0.95(0.90$ to 1.00$)$ \\
\hline 6 & UHRF1BP1 & rs11755393 & $A / G$ & 0.34 & 0.34 & 0.41 & 0.79 & & $1.01(0.95$ to 1.07$)$ \\
\hline 6 & ATG5 & rs6568431 & $\mathrm{C} / \mathrm{A}$ & 0.41 & 0.39 & 0.27 & 0.02 & 0.22 & $1.07(1.01$ to 1.13$)$ \\
\hline 7 & JAZF1 & rs864745 & $\mathrm{G} / \mathrm{A}$ & 0.49 & 0.49 & 0.98 & 0.20 & & $1.03(0.98$ to 1.08$)$ \\
\hline 8 & $B L K$ & rs2736340 & $\mathrm{C} / \mathrm{T}$ & 0.26 & 0.24 & 0.95 & $3.0 \times 10^{-4}$ & $3.3 \times 10^{-3}$ & $1.11(1.05$ to 1.17$)$ \\
\hline 11 & KIAA1542 & rs4963128 & $\mathrm{G} / \mathrm{A}$ & 0.32 & 0.34 & 0.96 & 0.02 & 0.22 & 0.93 (0.88 to 0.99$)$ \\
\hline 16 & ITGAM & rs9888739 & $\mathrm{C} / \mathrm{T}$ & 0.10 & 0.11 & 0.42 & 0.16 & & $0.94(0.85$ to 1.03$)$ \\
\hline 22 & UBE2L3 & rs5754217 & $\mathrm{G} / \mathrm{T}$ & 0.21 & 0.19 & 0.37 & $2.4 \times 10^{-3}$ & 0.026 & $1.11(1.04$ to 1.19$)$ \\
\hline
\end{tabular}

Linkage disequilibrium between the genotyped and reported variant (in brackets), when different: TNFSF4 (rs2205960): $\mathrm{r}^{2}=0.95 ;$ TNIP1 (rs7708392): $\mathrm{r}^{2}=0.90 ;$ PTTG1 (rs2431099): $\mathrm{r}^{2}=0.60 ;$ JAZF1 (rs849142): $\mathrm{r}^{2}=1 ;$ ITGAM (rs11860650): $\mathrm{r}^{2}=0.92$.

Chr, chromosome; $\mathrm{Cl}$, confidence interval; HWE, Hardy-Weinberg equilibrium; MAF, minor allele frequency; OR, odds ratio; RA, rheumatoid arthritis; SLE, systemic lupus erythematosus; SNP, single nucleotide polymorphism.

design or failed genotyping, resulting in 11 SNP markers being available for analysis (rs10489265 in TNFSF4, rs10516487 in BANK1, rs10036748 in TNIP1, rs2431697 in PTTG1, rs11755393 in UHRF1BP1, rs6568431 in ATG5, rs864745 in JAZF1, rs2736340 in BLK, rs4963128 in KIAA1542, rs9888739 in ITGAM and rs5754217 in UBE2L3).

Genotyping was undertaken using a Sequenom platform with iPlex chemistry according to manufacturer's instructions (http:// www.sequenom.com). Quality control analysis was undertaken such that only SNPs and samples that passed a $90 \%$ quality control threshold were subject to further statistical analysis. Control allele frequencies were tested to ensure that they conformed to Hardy-Weinberg expectations, and SNPs that deviated significantly from this were removed from further analysis.

\section{Analysis}

First, genotype frequencies were compared between RA cases and controls using the $\chi^{2}$ trend test implemented in PLINK software ${ }^{22}$ to determine whether individual SLE susceptibility loci were also associated with RA. Where data were available for SLE variants in independent RA samples, data from this study were added to the data already available and reanalysed to determine the best estimate of the effect size. p Values $<0.0045$ were regarded as significant after correcting for multiple testing (11 tests) applying the Bonferroni correction.

Second, pathway analysis was carried out using Ingenuity Pathway Analysis 8.6 (Ingenuity Systems, http://www. ingenuity.com) in order to explore whether SNPs uniquely associated with either RA or SLE identified characteristic pathways. Bioinformatic analysis, using the Ingenuity Pathways Analysis library, identified the pathways that were most supported by the dataset. Molecules from the dataset that were associated with a canonical pathway in Ingenuity's knowledge base were considered for the analysis. Fisher's exact test was used to calculate a $\mathrm{p}$ value determining the probability that the association between the genes in the dataset and the canonical pathway is explained by chance alone.

Third, analysis of carriage of SLE alleles in patients with RA was carried out using STATA version 9.2 to determine if there is an overall enrichment of SLE susceptibility variants in RA cases. A SLE loci carriage score was calculated by summing the number of SLE risk (coded as 1) and protection (coded as -1) alleles carried by each individual, and differences in the mean score between RA cases and controls was tested using the Wilcoxon rank-sum test.
Finally, in order to explore how much of the genetic susceptibility to RA could be accounted for by variants identified to date, the sibling recurrence risk ratio $(\lambda s)$ was calculated using the formula:

$\lambda s=\left(1+\frac{p q(\gamma-1)^{2}}{2(p+\gamma q)^{2}}\right)^{2}$

where $q$ is the risk allele frequency, $p=1-q$ and $\gamma$ is the genotype relative risk under the additive model.

\section{RESULTS}

For the BANK1, JAZF1 and BLK SNPs, data were available from a previous GWAS in a UK population including 2000 RA cases and 3000 controls. ${ }^{23}$ In addition, part of the cohort used in our study had been previously genotyped for UBE2L3. ${ }^{24}$ Therefore, non-overlapping samples from these studies were added to the current cohort for the analysis of the three markers. Control allele frequencies for all SNPs tested conformed to Hardy-Weinberg expectations (table 1). Four SNPs, rs6568431 mapping to the ATG5 locus, rs2736340 close to BLK, rs4963128 mapping to the KIAA1542 locus and rs5754217 in UBE2L3 showed nominal evidence for association $(p<0.05)$. After applying a Bonferroni correction for the number of SNPs tested, only the SNPs mapping to BLK and UBE2L3 retained statistically significant evidence for association. It should be noted that control allele frequencies were similar and the direction of association was the same at all four loci with that observed in the SLE studies, although effect sizes were smaller. Therefore, although non-significant after applying a Bonferroni correction, ATG5 and KIAA1542 remain interesting candidates for further investigation. Repeating the association analysis in the anti-CCP positive and negative subgroups of RA cases did not substantially alter the observed effect sizes (data not shown). A test of heterogeneity of odds ratios (ORs) showed that the effect sizes were similar among all RA, anti-CCP positive RA and anti-CCP negative RA subgroups for all analysed SNPs ( $>>0.05)$.

A meta-analysis including our UK cohort and the US cohort in which association of $B L K$ with RA was described for the first time showed a strong association between the rs2736340 SNP in the BLK locus and RA ( $\mathrm{p}=5.6 \times 10^{-11}, \mathrm{OR}=1.1495 \%$ CI 1.10 to 1.19). We also performed a pooled analysis for UBE2L3, expanding the validation cohort from the meta-analysis of Stahl et a ${ }^{24}$ with the UK non-overlapping samples genotyped in our study, 
and including data from GWAS. In this expanded analysis, the rs5754217 at the UBE2L3 locus now reaches genome-wide significance $\left(p=2.3 \times 10^{-10}, O R=1.1495 \%\right.$ CI 1.09 to 1.19$)$.

We next performed a pathway analysis including the loci that have shown evidence of association with both SLE and RA (HLA-DRB1, PTPN22, STAT4, TNFAIP3, FCGR2A, PRDM1, IRF5, PXK, BLK and UBE2L2). All the over-represented pathways are involved in the immune response, such as dendritic cell maturation, $\mathrm{T}$ helper (Th) cell differentiation or CTLA4 signalling in cytotoxic T lymphocytes (table 2). As is the case in the overlapping loci, genes exclusively associated with RA (AFF3, ANKRD55/IL6ST, C5orf13, CCL21, CCR6, CD2/CD58, CD28, CD40, CTLA4, IL2/IL21, IL2RA, IL2RB, KIF5A, PRKCQ, PTPRC, RBPJ, REL, SPRED2, TAGAP, TRAF1/C5 and TRAF6) are part of immune response pathways, with pathways related to Th cell activation being most over-represented. However, when analysing genes associated only with SLE (ATG5, BANK1, ITGAM, JAZF1, PHRF1, PTTG1, TNFSF4, TNIP1 and $U H R F 1 B P 1)$, a different pattern emerged. Four significantly overrepresented pathways were identified but each included only one gene and only one of them was immune response related (table 2).

The number of SLE-associated SNPs showing at least nominal evidence for association with RA was higher than would be expected by chance alone. We explored, therefore, the extent of the total burden of SLE susceptibility alleles in RA. For this analysis we included previously generated genotype data for the markers: rs7574865 in STAT4, rs2476601 in PTPN22 and rs5029937 in TNFAIP3; and from this study: rs10489265 in TNFSF4, rs10516487 in BANK1, rs10036748 in TNIP1, rs2431697 in PTTG1, rs11755393 in UHRF1BP1, rs6568431 in ATG5, rs864745 in JAZF1, rs2736340 in BLK, rs4963128 in KIAA1542, rs9888739 in ITGAM and rs5754217 in UBEL2L3. We found that the mean number of SLE risk alleles carried by patients with RA was significantly higher than that found in controls (2.9 vs 2.5 , $\mathrm{p}=9.1 \times 10^{-7}$ ).

Finally, we calculated the sibling recurrence risk ratio $(\lambda s)$ for the confirmed RA and SLE overlapping loci (PTPN22, STAT4, TNFAIP3, FCGR2A, PRDM1, IRF5, PXK, BLK and UBE2L2). We estimate that, after excluding $H L A-D R B 1$ alleles, these explain $5.8 \%$ of the genetic susceptibility to RA as a whole, while all the non-HLA confirmed RA loci identified to date ${ }^{24}$ are able to explain $10.7 \%$.

\section{DISCUSSION}

The findings confirm the association of the BLK and UBE2L3 loci with RA thus adding to the list of loci showing overlap between RA and SLE, which currently include HLA-DRB1, PTPN22, STAT4, TNFAIP3, FCGR2A, PRDM1, IRF5 and PXK. In addition, two loci, ATG5 and KIAA1542, showed nominal association with RA and the associated allele was the same as that previously reported for SLE, although the associations did not remain statistically significant after Bonferroni correction was applied. However, the effect sizes conferred by BLK, ATG5 and KIAA1542 were significantly higher for SLE than for RA (test of heterogeneity of ORs $\mathrm{p}$ values $=1 \times 10^{-6}, 3 \times 10^{-3}$ and $4 \times 10^{-3}$, respectively).

The finding of an association with $B L K$ confirms a study in a US population where this locus was also associated with RA. ${ }^{25}$ Interestingly, the $B L K$ locus has also shown association with RA in a Japanese population. ${ }^{26} B L K$ encodes a tyrosine kinase that is involved in the regulation of $\mathrm{B}$-cell activation. ${ }^{27} \mathrm{~B}$ cells have a key role in the pathogenesis of both RA and SLE through the production of autoantibodies, antigen presentation to $\mathrm{T}$ cells and cytokine production, and B-cell depletion has proved successful in the treatment of these diseases. ${ }^{28}$ UBE2L3 encodes an ubiquitin-conjugating enzyme involved in the regulation of interferon and TLR7/9 signalling pathways. ${ }^{4}$ Furthermore, this locus has been shown to be associated with coeliac disease. ${ }^{29}$ Therefore, BLK and UBE2L3 are promising candidate genes for both RA and SLE.

The lack of association in our population between RA and markers mapping to ITGAM and BANK1 is in accordance with a previous study in a Spanish population. ${ }^{30}$ However, the Spanish study failed to detect association with $B L K$, probably owing to lack of power to detect modest effect sizes. In this regard, our study had $>95 \%$ power to detect an association with similar effect sizes as those previously reported for SLE at the 5\% significance level for all the loci tested.

All the shared SLE-RA loci and RA-only loci are involved in important pathways for autoimmunity and inflammation. On the other hand, the burden of immune pathways seems to be lower for genes associated with SLE only. However, pathway analysis results should be considered with caution since, for most of the disease-associated loci, the true causal variant and therefore the gene responsible for the association has not yet been identified. In addition, the exact role of several associated genes has not been elucidated and our knowledge of the relationships between molecules is limited.

The degree of genetic overlap between RA and SLE is substantial, but it is difficult to assess and likely to be an underestimate, based on several factors. First, we have only analysed associated loci at genome-wide significance levels; however, additional SLE loci not yet satisfying this criterion have been identified and there are probably more to be discovered. Second, large sample sizes were included in the studies undertaken in SLE leading to the identification of variants with small effects, for which studies in RA may be underpowered to detect. Third, as only the SNP identified in the primary study is tested in the other diseases, it may be that a different variant in the same gene/region is responsible for risk in the second disease.

There are already examples of loci common to two or more autoimmune diseases for which the associated SNP and/or allele is not the same in each disease. For example, the SNPs at the TNFAIP3 region, associated with RA, SLE, type 1 diabetes, are different from those associated with coeliac disease and psoriasis. ${ }^{20} 31-36$ Another example is the R620W variant of the PTPN22 gene, which has been widely associated with many autoimmune diseases but not psoriasis, ${ }^{37} 38$ for which evidence of association of two different PTPN22 SNPs (rs1217414 and rs3789604) has been found and this has been independently replicated. ${ }^{39}{ }^{40}$ In addition, with regard to the JAZF1 locus, the variant associated with prostate cancer ${ }^{41}$ differs from that associated with SLE, ${ }^{10}$ type 2 diabetes ${ }^{42}$ and height variation. ${ }^{43}$ For these reasons, the actual overlap between the two diseases may be higher than estimated in this study.

A high degree of overlap between SLE and RA susceptibility loci might be expected as the two diseases show some clinical overlap in joint involvement, autoantibody production, systemic features and response to treatments such as B-cell depletion (rituximab). Indeed, there was a proposal put forward for an overlap syndrome of 'rhupus' as some patients develop features of both diseases. ${ }^{44}$ For example, the frequency of antinuclear antibody, the hallmark of SLE, is higher in patients with RA than the general population. ${ }^{45}$ Unfortunately, antinuclear 
Table 2 Over-represented $(p<0.05)$ canonical pathways in which RA and SLE overlapping loci, RA only loci and SLE only loci are involved

\begin{tabular}{|c|c|c|c|}
\hline Canonical pathway & Type of pathway & p Value & Genes in pathway \\
\hline \multicolumn{4}{|l|}{ SLE/RA overlap } \\
\hline Dendritic cell maturation & $\begin{array}{l}\text { Cellular immune response, cytokine signalling, pathogen-influenced } \\
\text { signalling }\end{array}$ & $1.4 \times 10^{-4}$ & STAT4, FCGR2A, HLA-DRB1 \\
\hline CTLA4 signalling in cytotoxic T lymphocytes & Cellular immune response & $1.8 \times 10^{-3}$ & HLA-DRB1, PTPN22 \\
\hline $\begin{array}{l}\text { Role of NFAT in regulation of the immune } \\
\text { response }\end{array}$ & $\begin{array}{l}\text { Cellular immune response, humoral immune response, intracellular } \\
\text { and second messenger signalling }\end{array}$ & $6.2 \times 10^{-3}$ & FCGR2A, HLA-DRB1 \\
\hline Antigen presentation pathway & Cellular immune response, humoral immune response & 0.03 & $H L A-D R B 1$ \\
\hline Allograft rejection signalling & Cellular immune response, disease specific pathway & 0.03 & HLA-DRB1 \\
\hline Autoimmune thyroid disease signalling & $\begin{array}{l}\text { Cellular immune response, disease specific pathway, humoral } \\
\text { immune response }\end{array}$ & 0.03 & HLA-DRB1 \\
\hline Graft-versus-host disease signalling & Cellular immune response, disease specific pathway & 0.03 & HLA-DRB1 \\
\hline TNFR1 signalling & Apoptosis, cytokine signalling & 0.03 & TNFAIP3 \\
\hline JAK/Stat signalling & $\begin{array}{l}\text { Apoptosis, cellular growth, proliferation and development, } \\
\text { intracellular and second messenger signalling }\end{array}$ & 0.04 & STAT4 \\
\hline \multicolumn{4}{|c|}{$e_{0}$} \\
\hline iCOS-iCOSL signalling in T helper cells & Cellular immune response & $9.8 \times 10^{-9}$ & PTPRC, CD28, PRKCQ, CD40, IL2RA, IL2RB \\
\hline T helper cell differentiation & Cellular immune response, cytokine signalling & $5.2 \times 10^{-8}$ & IL21, IL6ST, CD28, CD40, IL2RA \\
\hline Altered T-cell and B-cell signalling in RA & Cellular immune response, disease-specific pathways & $6.6 \times 10^{-6}$ & IL21, CD28, CD40, CCL21 \\
\hline T-cell receptor signalling & Cellular immune response & $1.3 \times 10^{-5}$ & PTPRC, CD28, PRKCQ, CTLA4 \\
\hline IL-12 signalling and production in macrophages & Cellular immune response, cytokine signalling & $2.0 \times 10^{-5}$ & TRAF6, PRKCQ, CD40, REL \\
\hline CD28 signalling in T helper cells & Cellular immune response & $2.7 \times 10^{-5}$ & PTPRC, CD28, PRKCQ, CTLA4 \\
\hline CD40 signalling & Cellular immune response, humoral immune response & $1.0 \times 10^{-4}$ & TRAF6, CD40, TRAF1 \\
\hline $\begin{array}{l}\text { Cross-talk between dendritic cells and natural } \\
\text { killer cells }\end{array}$ & Cellular immune response & $3.7 \times 10^{-4}$ & $C D 28, C D 40, I L 2 R B$ \\
\hline B cell development & $\begin{array}{l}\text { Cellular growth, proliferation and development, humoral immune } \\
\text { response }\end{array}$ & $9.3 \times 10^{-4}$ & PTPRC, CD40 \\
\hline B-cell activating factor signalling & $\begin{array}{l}\text { Cellular growth, proliferation and development, humoral immune } \\
\text { response }\end{array}$ & $1.7 \times 10^{-3}$ & TRAF6, TRAF1 \\
\hline Allograft rejection signalling & Cellular immune response, disease-specific pathways & $1.8 \times 10^{-3}$ & CD28, CD40 \\
\hline Autoimmune thyroid disease signalling & $\begin{array}{l}\text { Cellular immune response, disease-specific pathways, humoral } \\
\text { immune response }\end{array}$ & $1.9 \times 10^{-3}$ & $C D 28, C D 40$ \\
\hline Primary immunodeficiency signalling & $\begin{array}{l}\text { Cellular immune response, disease-specific pathways, humoral } \\
\text { immune response }\end{array}$ & $2.3 \times 10^{-3}$ & PTPRC, CD40 \\
\hline IL-2 signalling & Cellular immune response, cytokine signalling & $3.1 \times 10^{-3}$ & IL2RA, IL2RB \\
\hline Lymphotoxin receptor signalling & Apoptosis & $3.2 \times 10^{-3}$ & TRAF6, TRAF1 \\
\hline $\begin{array}{l}\text { Activation of IRF by cytosolic pattern recognition } \\
\text { receptors }\end{array}$ & Cellular immune response & $4.1 \times 10^{-3}$ & TRAFG, CD40 \\
\hline Small cell lung cancer signalling & Cancer, disease-specific pathways & $5.5 \times 10^{-3}$ & TRAF6, TRAF1 \\
\hline $\begin{array}{l}\text { Communication between innate and adaptive } \\
\text { immune cells }\end{array}$ & Cellular immune response & $5.8 \times 10^{-3}$ & $\mathrm{CD} 28, \mathrm{CD} 40$ \\
\hline IL-6 signalling & Cellular immune response, cytokine signalling & $8.1 \times 10^{-3}$ & TRAF6, IL6ST \\
\hline CTLA4 aignalling in cytotoxic T lymphocytes & Cellular immune response & $8.7 \times 10^{-3}$ & CD28, CTLA4 \\
\hline Type I diabetes mellitus signalling & Apoptosis, disease-specific pathways, & 0.01 & TRAF6, CD28 \\
\hline PKC signalling in T lymphocytes & Cellular immune response & 0.01 & CD28, PRKCO \\
\hline Hepatic fibrosis/hepatic stellate cell activation & Disease-specific pathways & 0.02 & CD40, CCL21 \\
\hline Hepatic cholestasis & Disease-specific pathways & 0.02 & TRAF6, PRKCO \\
\hline B cell receptor signalling & Humoral immune response & 0.02 & PTPRC, PRKCQ \\
\hline IL-8 signalling & Cellular immune response, cytokine signalling & 0.03 & TRAF6, PRKCQ \\
\hline Acute phase response signalling & Cytokine signalling & 0.03 & TRAF6, IL6ST \\
\hline $\begin{array}{l}\text { Role of NFAT in regulation of the immune } \\
\text { response }\end{array}$ & $\begin{array}{l}\text { Cellular immune response, humoral immune response, intracellular } \\
\text { and second messenger signalling }\end{array}$ & 0.03 & CD28, PRKCO \\
\hline
\end{tabular}


Table 2 Continued

\begin{tabular}{|c|c|c|c|}
\hline Canonical pathway & Type of pathway & p Value & Genes in pathway \\
\hline TNFR2 signalling & Apoptosis, cytokine signalling & 0.04 & TRAF1 \\
\hline 4-1BB signalling in T lymphocytes & Cellular immune response & 0.04 & TRAF1 \\
\hline \multicolumn{4}{|l|}{ SLE only } \\
\hline Mitotic roles of polo-like kinase & Cell cycle regulation & 0.035 & PTTG1 \\
\hline Angiopoietin signalling & $\begin{array}{l}\text { Cardiovascular signalling, cellular growth, proliferation and } \\
\text { development, growth factor signalling }\end{array}$ & 0.041 & TNIP1 \\
\hline Ubiquinone biosynthesis & Metabolism of cofactors and vitamins & 0.045 & UHRF1BP1 \\
\hline Caveolar-mediated endocytosis signalling & $\begin{array}{l}\text { Cellular immune response, organismal growth and development, } \\
\text { pathogen-influenced signalling }\end{array}$ & 0.046 & ITGAM \\
\hline
\end{tabular}

NFAT, nuclear factor of activated T cells; RA, rheumatoid arthritis; SLE, systemic lupus erythematosus.

antibody data were not available for the RA samples tested here so we were not able to explore whether the associations seen were greater in the antinuclear antibody-positive subgroup.

In conclusion, this study has shown that a high degree of overlap exists between RA and SLE and raises the possibility that shared phenotypic characteristics may be a result of shared genetic susceptibility loci implicated in overlapping inflammatory pathways.

Acknowledgements IT support was provided by Mark Lay, statistical advice from Mark Lunt and technical support by Paul Gilbert.

Funding This work was supported by the Arthritis Research UK (grant reference number 17552). The authors are grateful to the NIHR Manchester Biomedical Research Centre, the Wellcome Trust Case Control Consortium, NIHR-Leeds Musculoskeletal Biomedical Research Unit, the NIHR Oxford Musculoskeletal Biomedical Research Unit and the NIHR Oxford Biomedical Research Centre for support. GO is funded by the European Union (Marie Curie IEF Fellowship PIEF-GA2009-235662).

\section{Competing interests None.}

Patient consent Obtained.

Ethics approval This study was conducted with the approval of the MREC 99/8/84.

Provenance and peer review Not commissioned; externally peer reviewed.

\section{UKRAG Consortium}

University of Manchester'1: Stephen Eyre, Anne Hinks, Laura J Gibbons, John Bowes, Edward Flynn, Paul Martin, Xiayi Ke, Rachelle Donn, Wendy Thomson, Anne Barton, Jane Worthington.

University of Leeds': YEAR consortium, Stephen Martin, James I Robinson, Ann W Morgan, Paul Emery

University of Sheffield ${ }^{3}$ : Anthony G. Wilson

University of London ${ }^{4}$ : Sophia Steer

University of Aberdeen ${ }^{5}$ : Lynne Hocking, David M Reid

University of Oxford6: Pille Harrison, Paul Wordsworth

${ }^{1}$ Arthritis Research UK-Epidemiology Unit, Stopford Building, The University of Manchester, Manchester, UK

${ }^{2}$ Leeds Institute of Molecular Medicine, Section of Musculoskeletal Disease, University of Leeds, Leeds LS9 7TF, UK

${ }^{3}$ School of Medicine and Biomedical Sciences, The University of Sheffield, Sheffield S10 2JF, UK.

${ }^{4}$ Clinical and Academic Rheumatology, Kings College Hospital NHS Foundation Trust, Denmark Hill, London SE5 9RS, UK

${ }^{5}$ Bone Research Group, Department of Medicine and Therapeutics, University of Aberdeen AB25 2ZD, UK

${ }^{6}$ University of Oxford Institute of Musculoskeletal Sciences, Botnar Research Centre, Oxford OX3 7LD, UK

\section{REFERENCES}

1. Klareskog L, Catrina Al, Paget S. Rheumatoid arthritis. Lancet 2009;373:659-72.

2. Rahman A, Isenberg DA. Systemic lupus erythematosus. N Eng/ J Med 2008;358:929-39.

3. Orozco G, Barton A. Update on the genetic risk factors for rheumatoid arthritis. Expert Rev Clin Immunol 2010;6:61-75.

4. Graham RR, Hom G, Ortmann W, et al. Review of recent genome-wide association scans in lupus. J Intern Med 2009;265:680-8.

5. Hemminki K, Li X, Sundquist J, et al. Familial associations of rheumatoid arthritis with autoimmune diseases and related conditions. Arthritis Rheum 2009;60:661-8.
6. Hemminki K, Li X, Sundquist K, et al. Shared familial aggregation of susceptibility to autoimmune diseases. Arthritis Rheum 2009;60:2845-7.

7. Harley JB, Alarcón-Riquelme ME, Criswell LA, et al. Genome-wide association scan in women with systemic lupus erythematosus identifies susceptibility variants in ITGAM, PXK, KIAA1542 and other loci. Nat Genet 2008;40:204-10.

8. Hom G, Graham RR, Modrek B, et al. Association of systemic lupus erythematosus with C8orf13-BLK and ITGAM-ITGAX. N Engl J Med 2008;358:900-9

9. Kozyrev SV, Abelson AK, Wojcik J, et al. Functional variants in the B-cell gene BANK1 are associated with systemic lupus erythematosus. Nat Genet 2008:40:211-6

10. Gateva V, Sandling JK, Hom G, et al. A large-scale replication study identifies TNIP1, PRDM1, JAZF1, UHRF1BP1 and IL10 as risk loci for systemic lupus erythematosus. Nat Genet 2009;41:1228-33.

11. Barton A, Eyre $S$, Ke X, et al. Identification of AF4/FMR2 family, member 3 (AFF3) as a novel rheumatoid arthritis susceptibility locus and confirmation of two further pan-autoimmune susceptibility genes. Hum Mol Genet 2009;18:2518-22.

12. Coenen MJ, Trynka G, Heskamp S, et al. Common and different genetic background for rheumatoid arthritis and coeliac disease. Hum Mol Genet 2009;18:4195-203.

13. Smyth DJ, Plagnol V, Walker NM, et al. Shared and distinct genetic variants in type 1 diabetes and celiac disease. N Eng/ J Med 2008;359:2767-77.

14. Zhernakova A, Alizadeh BZ, Bevova M, et al. Novel association in chromosome $4 q 27$ region with rheumatoid arthritis and confirmation of type 1 diabetes point to a general risk locus for autoimmune diseases. Am J Hum Genet 2007; 81:1284-8

15. Orozco G, Hinks A, Eyre S, et al. Combined effects of three independent SNPs greatly increase the risk estimate for RA at 6q23. Hum Mol Genet 2009;18:2693-9.

16. Arnett FC, Edworthy SM, Bloch DA, et al. The American Rheumatism Association 1987 revised criteria for the classification of rheumatoid arthritis. Arthritis Rheum 1988;31:315-24.

17. MacGregor AJ, Bamber S, Silman AJ. A comparison of the performance of different methods of disease classification for rheumatoid arthritis. Results of an analysis from a nationwide twin study. J Rheumatol 1994;21:1420-6.

18. Barton A, Thomson W, Ke X, et al. Re-evaluation of putative rheumatoid arthritis susceptibility genes in the post-genome wide association study era and hypothesis of a key pathway underlying susceptibility. Hum Mol Genet 2008;17:2274-9.

19. Hinks A, Barton A, John S, et al. Association between the PTPN22 gene and rheumatoid arthritis and juvenile idiopathic arthritis in a UK population: further support that PTPN22 is an autoimmunity gene. Arthritis Rheum 2005:52:1694-9.

20. Thomson W, Barton A, Ke X, et al. Rheumatoid arthritis association at 6q23. Nat Genet 2007:39:1431-3.

21. Raychaudhuri S, Thomson BP, Remmers EF, et al. Genetic variants at CD28, PRDM1 and CD2/CD58 are associated with rheumatoid arthritis risk. Nat Genet 2009;41:1313-8.

22. Purcell S, Neale B, Todd-Brown K, et al. PLINK: a tool set for wholegenome association and population-based linkage analyses. Am J Hum Genet 2007;81:559-75.

23. Wellcome Trust Case Control Consortium. Genome-wide association study of 14,000 cases of seven common diseases and 3,000 shared controls. Nature 2007:447:661-78.

24. Stahl EA, Raychaudhuri S, Remmers EF, et al. Genome-wide association study meta-analysis identifies seven new rheumatoid arthritis risk loci. Nat Genet 2010;42:508-14.

25. Gregersen PK, Amos Cl, Lee AT, et al. REL, encoding a member of the NF-kappaB family of transcription factors, is a newly defined risk locus for rheumatoid arthritis. Nat Genet 2009;41:820-3.

26. Ito I, Kawasaki A, Ito $\mathrm{S}$, et al. Replication of association between FAM167A(C8orf13)-BLK region and rheumatoid arthritis in a Japanese population. Ann Rheum Dis 2010;69:936-7.

27. Dymecki SM, Zwollo P, Zeller K, et al. Structure and developmental regulation of the B-lymphoid tyrosine kinase gene blk. J Biol Chem 1992;267:4815-23. 
28. Edwards JC, Cambridge G. B-cell targeting in rheumatoid arthritis and other autoimmune diseases. Nat Rev Immunol 2006;6:394-403.

29. Garner CP, Murray JA, Ding YC, et al. Replication of celiac disease UK genome-wide association study results in a US population. Hum Mol Genet 2009;18:4219-25.

30. Suarez-Gestal M, Calaza M, Dieguez-Gonzalez R, et al. Rheumatoid arthritis does not share most of the newly identified systemic lupus erythematosus genetic factors. Arthritis Rheum 2009;60:2558-64.

31. Plenge RM, Cotsapas C, Davies L, et al. Two independent alleles at $6 q 23$ associated with risk of rheumatoid arthritis. Nat Genet 2007;39:1477-82.

32. Graham RR, Cotsapas C, Davies L, et al. Genetic variants near TNFAIP3 on 6q23 are associated with systemic lupus erythematosus. Nat Genet 2008;40:1059-61.

33. Musone SL, Taylor KE, Lu TT, et al. Multiple polymorphisms in the TNFAIP3 region are independently associated with systemic lupus erythematosus. Nat Genet 2008;40:1062-4

34. Fung EY, Smyth DJ, Howson JM, et al. Analysis of 17 autoimmune diseaseassociated variants in type 1 diabetes identifies 6q23/TNFAIP3 as a susceptibility locus. Genes Immun 2009;10:188-91.

35. Trynka G, Zhernakova A, Romanos J, et al. Coeliac disease-associated risk variants in TNFAIP3 and REL implicate altered NF-kappaB signalling. Gut 2009:58:1078-83.

36. Nair RP, Duffin KC, Helms C, et al. Genome-wide scan reveals association of psoriasis with IL-23 and NF-kappaB pathways. Nat Genet 2009;41:199-204.
37. Vang T, Miletic AV, Bottini N, et al. Protein tyrosine phosphatase PTPN22 in human autoimmunity. Autoimmunity 2007;:40:453-61.

38. Criswell LA, Pfeiffer KA, Lum RF, et al. Analysis of families in the multiple autoimmune disease genetics consortium (MADGC) collection: the PTPN22 $620 \mathrm{~W}$ allele associates with multiple autoimmune phenotypes. Am J Hum Genet 2005; 76:561-71.

39. Smith RL, Warren RB, Eyre S, et al. Polymorphisms in the PTPN22 region are associated with psoriasis of early onset. Br J Dermatol 2008;158:962-8.

40. Orrú V, Tsai SJ, Rueda B, et al. A loss-of-function variant of PTPN22 is associated with reduced risk of systemic lupus erythematosus. Hum Mol Genet 2009; 18:569-79

41. Thomas G, Jacobs KB, Yeager M, et al. Multiple loci identified in a genome-wide association study of prostate cancer. Nat Genet 2008;40:310-5.

42. Zeggini E, Scott LJ, Saxena R, et al. Meta-analysis of genome-wide association data and large-scale replication identifies additional susceptibility loci for type 2 diabetes. Nat Genet 2008;40:638-45.

43. Johansson A, Marroni F, Hayward C, et al. Common variants in the JAZF1 gene associated with height identified by linkage and genome-wide association analysis. Hum Mol Genet 2009:18:373-80.

44. Panush RS, Edwards NL, Longley S, et al. 'Rhupus' syndrome. Arch Intern Med 1988:148:1633-6.

45. Bell DA, Alspaugh MA. Antibody to rheumatoid arthritis associated nuclear antigen (RANA) in familial rheumatoid arthritis. J Rheumatol 1984:11:277-81. 